\title{
Psychosocial mediators of perceived stigma and suicidal ideation among transgender women
}

\author{
Krishna Kiran Kota ${ }^{1}$, Laura F. Salazar ${ }^{1 *}$ (D, Rachel E. Culbreth² ${ }^{2}$ Richard A. Crosby ${ }^{3,4}$ and Jamal Jones ${ }^{1}$
}

\begin{abstract}
Background: Transgender women (TGW) in the U.S. experience high rates of stigma, depression, and elevated rates of suicide. This study examined correlates of suicidal ideation and estimated the conditional indirect effects of perceived stigma and psychosocial mediators on suicidal ideation.

Methods: Using a cross-sectional study design, TGW ( $N=92)$ were recruited through snowball sampling in Atlanta, Georgia. Structured interviews were conducted. Suicidal ideation was assessed by combining two variables that measured suicidal thoughts. Logistic regression models were performed to identify the potential risk and protective factors for suicidal ideation. We examined hypothesized psychosocial factors, including anxiety, depression, psychosocial impact of gender minority status, and substance use behaviors as potential mediators for the relationship between perceived stigma and suicidal ideation. All models were controlled for age, race, education, and homelessness.
\end{abstract}

Results: Suicidal ideation was reported by $33 \%(N=30)$ of the study participants. In multivariable analysis, suicidal ideation was associated with sexual abuse $(A O R=3.17,95 \% \mathrm{Cl}=1.10-9.30)$, anxiety ( $\mathrm{AOR}=1.74,95 \% \mathrm{Cl}=1.10-2.73$ ), family verbal abuse $(A O R=2.99,95 \% C l=1.10-8.40)$, stranger verbal abuse ( $A O R=3.21,95 \% C l=1.02-10.08)$, and psychosocial impact of gender minority status ( $A O R=3.42,95 \% \mathrm{Cl}=1.81-6.46$ ). Partner support was found to be the protective factor for suicidal ideation ( $A O R=0.34,95 \% \mathrm{Cl}=0.13-0.90)$. In the mediation analysis, the psychosocial impact of gender minority status mediated the relationship between perceived stigma and suicidal ideation. The estimated conditional indirect effect was $0.46,(95 \% \mathrm{Cl}=0.12-1.11)$.

Conclusion: Interventions that aim to reduce suicidal behaviors among TGW should address stigma, psychosocial impact of gender minority status, and different forms of violence and abuse.

Keywords: Transgender women, Suicidal ideation, Sexual abuse, Perceived stigma, Psychosocial impact of gender minority status

\section{Background}

Transgender is an umbrella term for individuals whose gender identity or gender expression differs from what is typically associated with the sex that they were assigned at birth. The transgender community includes individuals, who were assigned male at birth and identify as female, who were assigned female at birth and identify as male, and who identify their gender as outside the binary

\footnotetext{
* Correspondence: Isalazar1@gsu.edu

'Department of Health Policy \& Behavioral Science, School of Public Health, Georgia State University, PO Box 3984, Atlanta, GA 30302-3984, USA Full list of author information is available at the end of the article
}

categories of male or female [1-4]. In the United States, there are significant disparities in suicide risk based on gender identity. Transgender populations have elevated rates of suicidal ideation and suicide [5-7]. According to the US Trans Survey (USTS), attempted suicide was reported by $40 \%$ of transgender women (TGW) survey participants compared to $1.6 \%$ in the general population and $10.2 \%$ among Lesbian, Gay, and Bisexual (LGB) participants $[5,8]$. Similarly, other studies found that 32.4 to $45.8 \%$ of transgender study participants reported lifetime suicide attempts $[9,10]$. Suicidal ideation which is defined as "thinking about, considering, or planning

(c) The Author(s). 2020 Open Access This article is distributed under the terms of the Creative Commons Attribution 4.0 International License (http://creativecommons.org/licenses/by/4.0/), which permits unrestricted use, distribution, and 
for suicide" is also highly prevalent among TGW [11]. Multiple studies have found prevalence rates of suicidal ideation among TGW that range from 35.1 to $79.2 \%$ [9, $12,13]$. In one study, an alarming $78.1 \%$ of the participants reported suicidal ideation in the past year [14]. Adams et al. in 2017 conducted a meta-analysis and reported that, across the 23 studies that were conducted from 1997 to 2016, among the transgender population, the prevalence of lifetime suicidal ideation was reported by $55 \%$, and suicidal ideation in the past 12 months was reported by $51 \%$ [15]. The same study also found higher lifetime suicidality among TGW (51.7\%) compared to transgender men $(45.4 \%)$, gender non-conforming individuals $(30 \%)$, and cross-dressers $(25.6 \%)$. As would be expected, suicidal ideation among TGW has been strongly associated with a history of suicide attempts [11, 16-18], and is a strong predictor of future suicide attempts [19]. Together, these findings highlight the need to identify the correlates of suicidal ideation among TGW, which would help to inform interventions to prevent suicidal ideation and suicide attempts.

Among cisgender populations, that is, people whose gender identity and gender expression align with their assigned sex at birth [20], research has identified predictors of suicidal ideation, including substance abuse, experiences of violence, depression, anxiety, and other mental health issues [18, 21-24]. For TGW, in addition to these factors, psychosocial factors that are specific to TGW, including stigma and discrimination, microaggressions, experiences of abuse and violence, family rejection, and lack of social support, could contribute to disproportionate rates of suicidality [12, 13, 25-27]. According to the USTS, transgender participants experienced various forms of discrimination due to their gender identity [5, 28], including unemployment (30\%), being refused a home or apartment (23\%), verbal harassment (46\%), being denied equal treatment by a government agency or official (24\%), and mistreatment by police $(58 \%)$ [5, 8]. Several other studies have reported that TG individuals experience high levels of transgender-related discrimination and stigma [29]. For example, transgender individuals have elevated rates of being denied access to care, as well as experience verbal harassment and physical violence, when attempting to access doctors and hospitals, emergency rooms, and using ambulances/by Emergency Medical Technicians [5, 8].

The stigma and discrimination experienced due to their gender identity may be associated with several adverse health outcomes among TGW [30]. Stigma may be indirectly associated with poor mental health in TGW by restricting their access to healthcare, housing, and employment [31, 32]. There also is evidence of a direct association between stigma and stress and subsequent mental health problems [30, 33, 34]. Societal attitudes toward TGW and the discrimination experienced by
TGW on a daily basis may have a significant psychosocial impact on TGW and could be an important factor in explaining the mechanism that leads to mental health issues and negative health behaviors. For example, there is evidence that perceived stigma and discrimination are associated with anxiety and depressive symptoms [35, 36], distress [33], suicide attempts [25], and a host of other negative mental outcomes [35,37].

Minority stress theory states that sexual and gender minorities experience stressors, such as discrimination and stigma, that lead to increased levels of stress that can, in turn, deplete psychological resources (e.g., resilience, social support) and lead to poor overall mental and physical health outcomes [38]. In this theory, perceived stress is viewed as the mediator of the association between sexual/gender minority status and negative health outcomes. A complement to the minority stress theory is the psychological mediation framework, which postulates that sexual and gender minorities experience stigma-related stress that leads to certain intraand interpersonal psychological processes that can affect mental health [39]. Within this framework, perceived stress relates to gender minority status and is the main predictor of negative health outcomes but with psychological and psychosocial factors that explain the association. Several studies have applied these theories to explain the disproportionate rates of mental health issues among sexual and gender minorities [9, 14, 40-42].

There is evidence that psychological and psychosocial factors, such as substance use, depression, and anxiety, may explain the association between perceived stigma and suicidal ideation [13]. Substance use has been hypothesized as a potential negative coping mechanism for the stress associated with stigma and discrimination among transgender persons. Substance use has demonstrated strong associations with suicidal ideation and suicide attempts [43] and has found to be a statistically significant mediator between stigma and suicidality among transgender individuals [43]. Moreover, the depression and anxiety associated with experiencing stigma and discrimination may lead to suicidal ideation and suicide attempts $[8,13]$. Taken together, these psychosocial factors, including anxiety, depression, negative impact of gender minority status, and substance use behaviors could be hypothesized as mediators. In this study, our goal is to examine the relationship between perceived stigma by TGW, the psychosocial factors that include anxiety and depression, and the psychosocial impact of gender minority status, substance use behaviors, and suicidal ideation.

Evidence for the prevalence and correlates of suicidal ideation among TGW is found in the literature $[12,13$, 25-27], but there is limited research [9, 14, 27, 41] on the role of psychosocial factors and the underlying 
mechanisms associated with suicidal ideation. This critical research gap needs to be addressed, as the findings could inform researchers and policymakers in designing suicide prevention interventions for TGW in the United States. In this study, we aim to measure the prevalence of suicidal ideation and to identify the demographic and psychosocial correlates of suicidal ideation and the potential underlying pathways associated with suicidal ideation among TGW. The conceptual framework (Fig. 1) shows our hypothesized model of the psychosocial factors that explain the pathway between perceived stigma and suicidal ideation. The psychosocial factors include anxiety, depression, the psychosocial impact of gender minority status, and substance use behaviors.

\section{Methods}

\section{Participants and procedures}

In this cross-sectional study, multiple community-based outreach strategies were used to recruit a sample of TGW $(n=92)$ between the ages of 18 and 65 years who reside in Atlanta, GA. Venues that serve TGW and word-of-mouth recommendations from transgender advocates provided the primary methods of recruitment. These venues offer HIV prevention and care, housing, and counseling services to the TGW. The study was known as the Transgender Atlanta Personal Survey. Transgender advocates notified the study project director when they located a woman who was willing to be screened for study participation. In addition, the project was advertised through formal and informal communication channels via advocacy groups and Lesbian, Gay, Bisexual, and Transgender (LGBT) service organizations. The project director used print materials to provide their contact information. Data were collected from $\mathrm{Au}$ gust 2014 through June 2015.
TGW were screened to determine eligibility. The inclusion criteria were: (1) 18 to 65 years of age, (2) male sex assigned at birth, and (3) self-identifying as either female or transgender. All participants who were screened, except one individual who identified as "other" were eligible and consented to participate in the study. After providing written informed consent, women engaged in a face-to-face structured interview with a trained graduate research assistant. The training involved cultural-competency and the use of non-judgmental statements. Interview responses were recorded on a portable electronic tablet, using Qualtrics $\odot$ software (Provo, Utah). The Institutional Review Board of Georgia State University approved study protocols following a full board review.

\section{Measures}

The survey assessed sociodemographic characteristics, a broad range of theoretical contextual factors, and selfreported HIV status. In addtion, we assessed the prevalence of several trauma exposures, such as "ever experienced physical abuse by an intimate partner," "ever being a victim of sexual abuse," "ever experienced childhood sexual abuse," and psychosocial factors.

Perceived stigma was assessed by using four items adapted for TGW from the original scale developed for gay individuals $[44,45]$. These four items were a subscale that measured the TGW's perceptions of society's stigma or negative attitudes toward TGW. The items were: (1) "Society still punishes people for being transgender"; (2) "Most people have negative reactions to transgender people"; (3) "Discrimination against transgender people is still common"; and (4) "Only a few people discriminate against transgender people." Response options were presented on a 5-point Likert scale, ranging from $1=$ strongly disagree to $5=$ strongly agree.

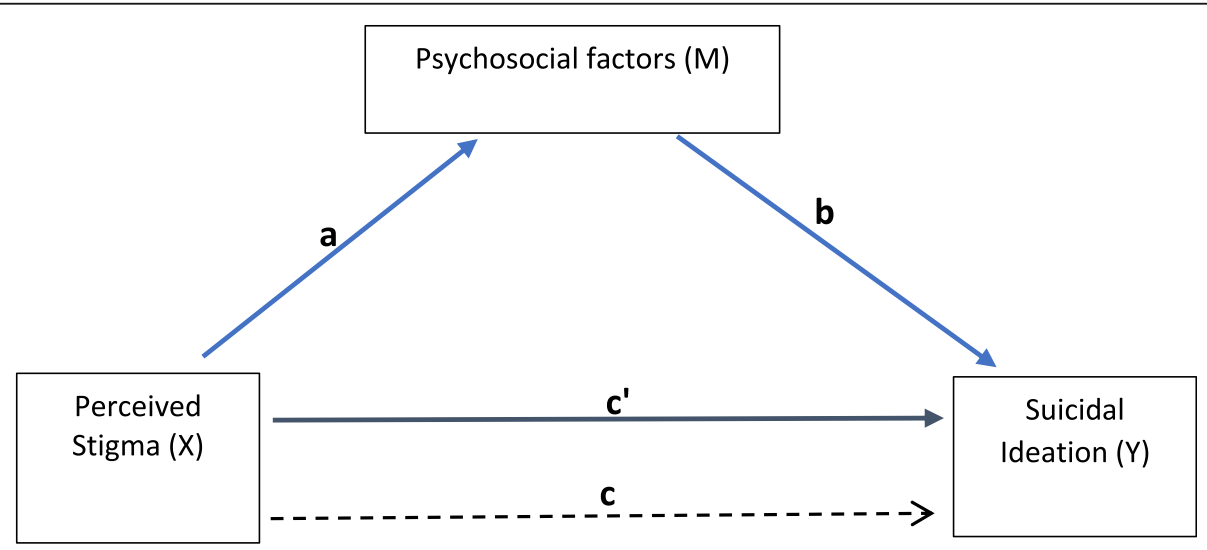

Fig. 1 Conceptual Diagram of the mediation models. Path c - Total effect of perceived stigma (X) on suicidal ideation (Y). path a - Effects of perceived stigma $(X)$ on psychosocial factors (Mediators $(M)$ : anxiety, depression, the psychosocial impact of gender minority status, excessive drinking, injection drug use, and non-injection drug use). path b - Effects of psychosocial factors (M) on suicidal ideation ( $Y$ ) controlling for perceived stigma $(X)$. path $c^{\prime}$ - Direct effect of perceived stigma $(X)$ on suicidal ideation $(Y)$ controlling for psychosocial factors (M) 
Item 4 was reverse coded. The mean of response scores for the four items were used for the analysis. Inter-item reliability was adequate (Cronbach's alpha $=0.73$ ).

The psychosocial impact of gender minority status was assessed using three items from a 4-item subscale developed by Sjoberg and colleagues [46]. The 4-item subscale is part of the longer Transgender Adaptation and Integration Measure and assessed four aspects of mental health related to transgender status. We used this subscale to measure psychosocial distress related to the unique experiences of TGW. The items were: (1) "I get depressed about my gender status"; (2) "My gender status interferes with my quality of life"; (3) "I have thought about suicide because of my gender status"; and (4) "Being transgender causes me relationship problems." Response options were provided on a 5-point Likert scale, ranging from $1=$ strongly disagree to $5=$ strongly agree. Given that our outcome was suicidal ideation, the third item was not included in the analyses. We used the average of response scores for the three items. Inter-item reliability was adequate (Cronbach's alpha $=0.71$ ).

Suicidal ideation was assessed by combining two items that measured suicidal thoughts. The items were: (1) "In the past 12 months, have you considered attempting suicide?" for which the response options were Yes/No; and (2) "I have thought about suicide because of my gender status," for which response options were provided on a 5-point Likert scale, ranging from $1=$ strongly disagree to $5=$ strongly agree. We dichotomized Item 2 by collapsing the responses of 4 (agree) and 5 (strongly agree) as "Yes" and all other responses as "No." Then, we created a new variable, "suicidal ideation," for the participants who responded "Yes" to either of the two items; these participants were considered as experiencing suicidal ideation and other participants, as not experiencing suicidal ideation.

Depression was measured using six items from the Brief Symptom Inventory [47]. This subscale is widely used as a psychological self-report symptom scale to measure depression. The items were: (1) "Feeling not interested in things"; (2) "Feeling lonely"; (3) "Feeling blue"; (4) "Feeling worthlessness"; (5) "Feeling hopeless about the future"; and (6) "Thoughts of ending your life." Response options for all items were on a 5-point Likert scale, ranging from $1=$ not at all to $5=$ extremely. We calculated the mean of these six items as the depression score.

Anxiety was measured using the 3-item subscale from the Brief Symptom Inventory. The items were: (1) "Experienced nervousness or shakiness inside"; (2) "Feeling tense or keyed up"; and (3) "Feeling so restless you couldn't sit still." Response options for all items were on a 5 -point Likert scale, ranging from $1=$ not at all to $5=-$ extremely. For the anxiety score, we calculated the mean of the three items.
Excessive drinking was measured by three items: (1) "In the past 30 days, on how many days did you drink any alcohol?"; (2) "On the days when you drank alcohol in the past 30 days, about how many drinks did you have on average?"; and (3) "In the past 30 days, how many times did you have 5 or more alcoholic drinks in one sitting?" Based on the Dietary Guidelines for Americans, 2015-2020 [48], participants who consumed 15 or more drinks during the prior week or consumed more than 5 or more drinks in one sitting were considered to evidence excessive drinking.

Non-injection drug use was measured by one item: "In the past 12 months, have you used any non-injection drugs, other than those prescribed for you?" Response options were Yes/No.

Injection drug use was measured by one item: "Have you ever in your life shot up or injected any drugs other than those prescribed for you? By drugs, I am referring to drugs such as heroin, meth - not hormones or silicone? By shooting up, we mean anytime you might have used drugs with a needle, either by mainlining, skin popping, or muscling." Response options were Yes/No.

Intimate partner violence is the experience of physical and emotional violence by a romantic or sexual partner in one's lifetime. This variable was measured by three items: (1) "In your lifetime, have you ever been physically abused by a romantic or sexual partner? By physical abuse, we mean a range of behaviors, from slapping, pushing, or shoving, to severe acts, such as being beaten, burned, or choked"; (2) "In your lifetime, have you ever been emotionally abused by a romantic or sexual partner? By 1 emotional abuse, we mean name-calling, or humiliating you, or trying to monitor and control or threaten you"; and (3) "Have you ever been physically abused or beaten by a romantic or sexual partner because of your gender identity or presentation?" Response options were Yes/No.

Sexual abuse is the experience of forced oral/anal sex in one's lifetime. This variable was measured by three items: (1) "In your lifetime, has someone ever made you perform oral sex?"; (2) "In your lifetime, has someone ever made you receive anal sex? By receiving anal sex, we mean they put their penis in your anus (you were the bottom)"; and (3) "In your lifetime, has someone ever made you perform anal sex? By performing anal sex, we mean they made you put your penis in their anus (you were the top)". Response options were Yes/No.

Child sexual abuse was measured by one item: "As a child (less than 16 years old), were you ever sexually abused?" Response options were Yes/No/I do not remember. HIV status was measured by one item, "What was the result of your most recent HIV test?" for which the response options were "negative"/ "positive"/ "I do not know my status". 


\section{Data analysis}

Descriptive statistics were computed among TGW who reported suicidal ideation and those who reported no suicidal ideation. To estimate bivariate associations, TGW with suicidal ideation were compared to TGW without suicidal ideation, using chi-square analyses and Fisher's exact test for categorical variables (i.e., education, race, homelessness, excessive drinking, noninjection, injection drug use, and HIV status). Wilcoxon rank-sum tests were used for continuous variables (i.e., age, perceived stigma, anxiety, depression, and psychosocial impact of gender minority status). Control variables were selected based on the literature and the presence of statistically significant differences in our bivariate analyses $(\mathrm{alpha}=0.10)$. Age, race, and education were statistically significantly different between both groups, and homelessness was associated with suicidal ideation among TGW in prior studies. To estimate the association of substance abuse behaviors, violence, abuse, HIV status, and other psychosocial factors with suicidal ideation, we conducted separate multivariable logistic regression models, adjusting for age, race, education, and homelessness.

We also examined the impact of perceived stigma on suicidal ideation through mediation analyses. A mediator is a variable that explains, or accounts for, the effect of the independent variable on the dependent variable [49, 50]. To examine the underlying mechanism between perceived stigma and suicidal ideation, we tested the role of six psychosocial factors as potential mediators to explain the effect of perceived stigma (Variable X) on suicidal ideation (Variable Y) (Fig. 1): anxiety, depression, the psychosocial impact of gender minority status, excessive drinking, injection drug use, and non-injection drug use (Variables M). Six separate mediation models were tested, one for each psychosocial factor. The effect of $\mathrm{X}$ on $\mathrm{Y}$ is the total effect (path c); the effect of $\mathrm{X}$ on $\mathrm{M}$ is indicated by path $a$; the effect of $\mathrm{M}$ on $\mathrm{Y}$ controlling for $\mathrm{X}$, is indicated by path $b$; and the direct effect of $\mathrm{X}$ on $\mathrm{Y}$, controlling for $M$, is path $c$ '. The indirect effect is the product of path $a$ and path $b$, which is path $a b$. The equation $\left(c=c^{\prime}+a b\right)$, which indicates the total effect is equal to direct and indirect effects, does not hold true due to the use of logistic regression. By standardizing the coefficients expressed on a log-odds metric (multiplied by the standard deviation of the predictor variable and divided by standard deviation of outcome variable), however, $c$ would be approximately equal to $c^{\prime}+a b$ [49]. Using PROCESS macros v3.3 by Andrew F. Hayes [51], we tested the psychosocial impact of gender minority status, anxiety, and depression as mediators, and the point estimates for path $a$, path $b$, path $c$, and path $c$ were generated. For testing the dichotomous mediators (excessive drinking, injection drug use and non-injection drug use) we used the INDIRECT macro [50]. Bootstrapping $(N=5000)$ was used to construct confidence intervals (CIs) for the indirect effect (path $a b$ ) to determine statistically significant mediators. The Statistical Package for Social Sciences (SPSS), version 25.0, (IBM, Chicago, IL), was used for all analyses.

\section{Results}

Among the total study sample of TGW $(N=92)$, the prevalence of suicidal ideation was 33\% $(n=30)$. The average age of participants was 35 years, $51 \%$ were homeless, $60 \%$ of the participants who are aware of their HIV status reported as HIV positive, $50 \%$ had more than a high-school education, and $84 \%$ identified as Black or African American (Table 1). Of the study participants, sexual abuse was reported by $62 \%$, and child sex abuse was reported by $52 \%$. Substance abuse behaviors included excessive drinking (21\%), injection drug use (15\%), and non-injection drug use (34\%). Among the total study sample, the mean score for the perceived stigma measure was 4.03 ( $\mathrm{SD}=0.66$, range: $1-5)$, mean score of anxiety was 2.11 ( $\mathrm{SD}=1.14$, range: $1-5)$, mean score of depression was $2.01(\mathrm{SD}=0.85$, range: $1-5)$, and mean score on the psychosocial impact of gender minority status measure was 2.63 ( $\mathrm{SD}=0.94$, range: $1-5)$. Family verbal abuse and stranger verbal abuse were reported by $53 \%$ and $63 \%$ of the study participants, respectively. As shown in Table 1, significant differences between TGW who experiencd sucidial ideation and those who had not were found on a host of demographic and psychosocial variables.

In the multivariable analysis (Table 2), suicidal ideation was associated with sexual abuse (AOR: 3.17, 95\% CI: 1.10, 9.30), higher anxiety scores (AOR: 1.74; 95\% CI: $1.10,2.73)$, psychosocial impact of gender minority status (AOR: 3.42, 95\% CI: 1.81, 6.46), family verbal abuse (AOR: 2.99; 95\% CI: 1.10, 8.40), stranger verbal abuse (AOR: 3.21; 95\% CI: 1.02, 10.08), and partner support (AOR: 0.34; 95\% CI: 0.13, 0.90). Depression, perceived stigma, non-injection drug use, intimate partner violence, child sex abuse, and HIV status were no longer associated with suicidal ideation in the multivariable analyses.

In the mediation analyses (Table 3), results showed that perceived stigma had a significant direct effect on the psychosocial impact of gender minority status (path a), $(0.38, \mathrm{SE}=0.15,95 \% \mathrm{CI}=0.08 .0 .68)$. The psychosocial impact of gender minority status also was significantly associated with suicidal ideation (path $b)(1.19, \mathrm{SE}=$ $0.33,95 \% \mathrm{CI}=0.53,1.85)$, and there was a significant indirect effect (path $a b$ ) of the psychosocial impact of gender minority status on the association between perceived stigma and suicidal ideation $(0.46, \mathrm{SE}=0.26,95 \% \mathrm{CI}$ : $0.12,1.11)$. The other psychosocial mediator variables, 
Table 1 Descriptive statistics by suicidal ideation among transwomen residing in Atlanta, Georgia

\begin{tabular}{|c|c|c|c|}
\hline \multirow[t]{2}{*}{ Characteristic } & \multirow{2}{*}{$\begin{array}{l}\text { Suicidal } \\
\text { Ideation } \\
\text { (Yes), N }=30 \\
(33 \%)\end{array}$} & \multirow{2}{*}{$\begin{array}{l}\text { Suicidal } \\
\text { Ideation } \\
(\mathrm{No}), N=62 \\
(67 \%)\end{array}$} & \multirow{2}{*}{$\begin{array}{l}\text { Total } \\
N=92 \\
(\%)\end{array}$} \\
\hline & & & \\
\hline Age, Median (IQR)** & $32(11)$ & $36(11)$ & $35(11)$ \\
\hline \multicolumn{4}{|l|}{ Education* } \\
\hline High school or less & $11(37)$ & $35(57)$ & $46(50)$ \\
\hline College or higher & $19(63)$ & $27(43)$ & $46(50)$ \\
\hline \multicolumn{4}{|l|}{ Race $^{* *}$} \\
\hline African American/ Black & $18(60)$ & $59(95)$ & $77(84)$ \\
\hline Caucasian/ White & $8(27)$ & $2(3)$ & $10(11)$ \\
\hline Others & $4(13)$ & $1(2)$ & $5(5)$ \\
\hline \multicolumn{4}{|l|}{ Homeless $^{a}$} \\
\hline Yes & $15(50)$ & $31(51)$ & $46(51)$ \\
\hline No & $15(50)$ & $30(49)$ & $45(49)$ \\
\hline \multicolumn{4}{|l|}{ Non-injection drug use $\mathrm{e}^{* a}$} \\
\hline Yes & $15(52)$ & $16(26)$ & $31(34)$ \\
\hline No & $14(48)$ & $46(74)$ & $60(66)$ \\
\hline \multicolumn{4}{|l|}{ Injection drug use } \\
\hline Yes & $4(13)$ & $10(16)$ & $14(15)$ \\
\hline No & $26(87)$ & $52(84)$ & $78(85)$ \\
\hline \multicolumn{4}{|l|}{ Excessive drinking } \\
\hline Yes & $9(30)$ & $10(16)$ & $19(21)$ \\
\hline No & $21(70)$ & $52(84)$ & $73(79)$ \\
\hline \multicolumn{4}{|c|}{ Sexual abuse by any perpetrator (lifetime)** } \\
\hline Yes & $23(77)$ & $34(55)$ & $57(62)$ \\
\hline No & $7(23)$ & $28(45)$ & $35(38)$ \\
\hline \multicolumn{4}{|c|}{ Intimate partner violence (lifetime) ${ }^{* *}$} \\
\hline Yes & $28(93)$ & $45(73)$ & $73(79)$ \\
\hline No & $2(7)$ & $17(27)$ & $19(21)$ \\
\hline \multicolumn{4}{|c|}{ Child Sex Abuse (less than 16 years old)*a } \\
\hline Yes & $19(66)$ & $28(46)$ & $47(52)$ \\
\hline No & $10(34)$ & $33(54)$ & $43(48)$ \\
\hline \multicolumn{4}{|l|}{ Mean (Standard Deviation) } \\
\hline $\begin{array}{l}\text { Anxiety (3 items) } \\
\text { (Range } 1 \text { to } 5)^{* *}\end{array}$ & $2.54(1.38)$ & $1.90(0.96)$ & $\begin{array}{l}2.11 \\
(1.14)\end{array}$ \\
\hline $\begin{array}{l}\text { Depression (6 items) (Range } \\
1 \text { to } 5)^{* *}\end{array}$ & $2.24(0.79)$ & $1.90(0.86)$ & $\begin{array}{l}2.01 \\
(0.85)\end{array}$ \\
\hline $\begin{array}{l}\text { Perceived stigma ( } 4 \\
\text { items) (Range } 1 \text { to } 5)^{* *}\end{array}$ & $4.23(0.63)$ & $3.92(0.66)$ & $\begin{array}{l}4.03 \\
(0.66)\end{array}$ \\
\hline $\begin{array}{l}\text { Psychosocial impact of } \\
\text { gender minority status } \\
\text { ( } 3 \text { items) (Range } 1 \text { to } 5 \text { ) ** }\end{array}$ & $3.26(0.97)$ & $2.33(0.77)$ & $\begin{array}{l}2.63 \\
(0.94)\end{array}$ \\
\hline \multicolumn{4}{|l|}{ Family verbal abuse ${ }^{* *}$} \\
\hline Yes & $21(70)$ & $28(45)$ & $49(53)$ \\
\hline No & $9(30)$ & $34(55)$ & $43(47)$ \\
\hline
\end{tabular}

Stranger verbal abuse ${ }^{* *}$
Table 1 Descriptive statistics by suicidal ideation among transwomen residing in Atlanta, Georgia (Continued)

\begin{tabular}{|c|c|c|c|}
\hline \multirow[t]{2}{*}{ Characteristic } & \multirow{2}{*}{$\begin{array}{l}\text { Suicidal } \\
\text { Ideation } \\
\text { (Yes), N= }=30 \\
(33 \%)\end{array}$} & \multirow{2}{*}{$\begin{array}{l}\text { Suicidal } \\
\text { Ideation } \\
(\mathrm{No}), N=62 \\
(67 \%)\end{array}$} & \multirow{2}{*}{$\begin{array}{l}\text { Total } \\
N=92 \\
(\%)\end{array}$} \\
\hline & & & \\
\hline Yes & $25(83)$ & $38(61)$ & $63(68)$ \\
\hline No & $5(17)$ & $24(39)$ & $29(32)$ \\
\hline \multicolumn{4}{|l|}{$H I V^{* *, a}$} \\
\hline Positive & $10(40)$ & $40(60)$ & $50(60)$ \\
\hline Negative & $15(60)$ & $18(31)$ & $33(40)$ \\
\hline \multicolumn{4}{|c|}{ Partner Support** } \\
\hline Yes & $15(50)$ & $48(77)$ & $63(68)$ \\
\hline No & $15(50)$ & $14(23)$ & $29(32)$ \\
\hline
\end{tabular}

not add up due to missing data

anxiety, depression, excessive drinking, injection drug use, and non-injection drug use, were not significant mediators. The mediation effect size was estimated by calculating the ratio of (path ab/path c) [52]. The psychosocial impact of gender minority status mediated $77 \%$ of the effect of perceived stigma on suicidal ideation.

\section{Discussion}

In this study, we sought to determine the correlates for suicidal ideation among TGW and examined the mediation pathways that explain the underlying relationships. In our sample, the prevalence of suicidal ideation was $33 \%$, within the range reported by other studies $[13,53]$. We found that psychosocial factors, including anxiety, perceived stigma of being transgender, the psychosocial impact of gender minority status, experiencing sexual abuse, family verbal abuse, and stranger verbal abuse were significantly associated with higher odds of suicidal ideation. Partner support was found to be a protective factor.

Using the postulates of minority stress theory and the psychological mediation framework, we investigated whether certain psychosocial factors explained the associations between perceived stigma and suicidal ideation. We found that the psychosocial impact of gender minority status was a statistically significant mediator in our sample, indicating that the effect of perceived stigma on suicidal ideation may be explained by this pathway. The other psychosocial mediators examined, depression, anxiety, and substance use behaviors, were not statistically significant, which is contrary to the findings of previous research [43].

The risk factors that we found to be significantly associated with suicidal ideation are in keeping with those of other studies that reported a lack of or low social support [7, 13, 54], sexual abuse and gender-based 
Table 2 Associations of suicidal ideation with risk factors among transwomen

\begin{tabular}{|c|c|}
\hline Characteristic & Adjusted OR $(95 \% \mathrm{Cl})$ \\
\hline \multicolumn{2}{|l|}{ Non-injection drug use } \\
\hline Yes & $2.30(0.81-6.53)$ \\
\hline No & 1.00 \\
\hline \multicolumn{2}{|l|}{ Injection drug use } \\
\hline Yes & $1.28(0.31-5.23)$ \\
\hline No & 1.00 \\
\hline \multicolumn{2}{|l|}{ Excessive drinking } \\
\hline Yes & $2.07(0.58-7.35)$ \\
\hline No & 1.00 \\
\hline \multicolumn{2}{|l|}{ Sexual abuse } \\
\hline Yes & $3.17(1.10-9.30) *$ \\
\hline No & 1.00 \\
\hline \multicolumn{2}{|l|}{ Intimate partner violence } \\
\hline Yes & $4.59(0.89-23.66)$ \\
\hline No & 1.00 \\
\hline \multicolumn{2}{|l|}{ Child Sex Abuse } \\
\hline Yes & $1.91(0.72-5.12)$ \\
\hline No & 1.00 \\
\hline Anxiety & $1.74(1.10-2.73) *$ \\
\hline Depression & $1.54(0.83-2.80)$ \\
\hline Perceived stigma & $1.75(0.81-3.76)$ \\
\hline Psychosocial impact of gender minority status & $3.42(1.81-6.46)^{*}$ \\
\hline \multicolumn{2}{|l|}{ Family verbal abuse } \\
\hline Yes & $2.99(1.10-8.40)^{*}$ \\
\hline No & 1.00 \\
\hline \multicolumn{2}{|l|}{ Stranger verbal abuse } \\
\hline Yes & $3.21(1.02-10.08)^{*}$ \\
\hline No & 1.00 \\
\hline \multicolumn{2}{|l|}{ HIV status } \\
\hline Positive & $0.37(0.13-1.10)$ \\
\hline Negative & 1.00 \\
\hline \multicolumn{2}{|l|}{ Partner Support } \\
\hline Yes & $0.34(0.13-0.90)^{*}$ \\
\hline No & 1.00 \\
\hline
\end{tabular}

${ }^{a}$ Adjusted for age, race, education, and homelessness. * $p$ - value $<0.05$, OR Odds Ratio, $\mathrm{Cl}$ - Confidence Intervals

discrimination [25], family verbal abuse [12], stigma and discrimination [26], and the psychosocial impact of gender minority status and internalized transphobia [10]. Taken together, these societal-level risk factors suggest that TGW are at risk for a multitude of traumatic experiences that have severe mental health sequelae. Until there is a shift in societal attitudes and norms, TGW could benefit from public health interventions, such as mind-body programs [55] that enhance resiliency and improve coping, or online-eHealth interventions [56] that provide a safe space to improve skills and receive support. At a minimum, mental health professionals and social service providers who work with this population should be sensitive to the abuse history and mental health needs of the TGW with whom they work.

As would be expected, interpersonal factors, such as experiencing sexual abuse; psychosocial factors, such as anxiety and depression; and trans-specific factors, such as perceived stigma, family and stranger verbal abuse due to gender identity; and the psychosocial impact of gender minority status predicted the likelihood of suicidal ideation among TGW. We also found that partner support was a protective factor among TGW, similar to other studies that found a reduced risk of suicidal ideation among the TGW with higher levels of social support [13], indicating the vital role of a support system for TGW. These findings suggest that interpersonal, trans-specific, and psychosocial factors should be the focus in the development of suicide prevention interventions.

We found that the construct, the psychosocial impact of gender minority status, which measures the psychosocial distress experienced by TGW related to their unique gender identity, was significantly associated with suicidal ideation. The psychosocial impact of gender minority status is different from the other general forms of distress, such as anxiety, psychological distress, and depression [46], and represents the psychological impact of stigma and discrimination based on gender identity. This result indicates that this specific form of distress related to TGW is a significant predictor of suicidal ideation. Structural interventions to change the policies and laws to combat the stigma and discrimination against TGW are necessary.

We also found that the psychosocial impact of gender minority status was a statistically significant mediator that partially explains the relationship between perceived stigma and suicidal ideation. This finding was similar to the studies that: (a) reported that internalized transnegativity (sometimes referred to as internalized transphobia) mediated the relationship between distal stressors (such as anti-trans stigma, discrimination and victimization) and suicidal ideation [40], and (b) applied the minority stress theory to TGW and found that, internal stressors (internalized transphobia) mediated the relationship between external distal stressors (anti-trans discrimination, stigma, and victimization) and suicidal ideation [9]. The model used in our study, however, was conceptualized differently from the frameworks applied in these previous studies. In our model, perceived stigma tapped into TGW's perceptions of the prejudice and discrimination in their community. In this context, perceived stigma could be viewed as a proxy for distal 
Table 3 Summary of mediation effects of psychosocial factors between perceived stigma and suicidal ideation

\begin{tabular}{|c|c|c|c|c|}
\hline Mediator & path a, SE, 95\% Cl & path b, SE, 95\% Cl & path c', SE, 95\% Cl & path ab, SE, 95\% Cl \\
\hline $\begin{array}{l}\text { Psychosocial impact of gender minority } \\
\text { status }\end{array}$ & $0.38 * 0.15,0.08-0.68$ & $1.19^{*}, 0.33,0.53-1.85$ & $\begin{array}{l}0.12,0.45,-0.75- \\
1.00 .\end{array}$ & $0.46^{*}, 0.26,0.12-1.11$ \\
\hline Anxiety & $0.36,0.18,-0.005-0.72$ & $0.48^{*}, 0.23,0.02-0.93$ & $\begin{array}{l}0.42,0.41,-0.38- \\
1.23\end{array}$ & $0.17,0.16,-0.02-0.60$ \\
\hline Depression & $0.17,0.14,-0.11-0.45$ & $0.40,0.28,-0.16-0.96$ & $\begin{array}{l}0.55,0.40,-0.24- \\
1.34\end{array}$ & $0.06,0.09,-0.11-0.28$ \\
\hline Excessive drinking & $-0.04,0.06,-0.15-0.07$ & $0.84,0.60,-0.33-2.01$ & $\begin{array}{l}0.62,0.40,-0.16- \\
1.40\end{array}$ & $-0.03,0.08,-0.33-0.06$ \\
\hline Injection drug use & $\begin{array}{l}-0.02,0.05,-0.11- \\
0.07\end{array}$ & $0.30,0.72,-1.11-1.71$ & $\begin{array}{l}0.57,0.39,-0.19- \\
1.33\end{array}$ & $\begin{array}{l}-0.006,0.18,-0.25- \\
0.09\end{array}$ \\
\hline Non-injection drug use & $0.05,0.07,-0.09-0.19$ & $\begin{array}{l}-1.01,0.55,-2.09- \\
0.07\end{array}$ & $\begin{array}{l}0.81,0.42,-0.01- \\
1.63\end{array}$ & $-0.05,0.09,-0.36-0.06$ \\
\hline
\end{tabular}

Models were adjusted for age, homelessness, education, and race. All effects are on a log-odds metric

${ }^{*} p<0.05$, SE Standard Error, $\mathrm{Cl}$ Confidence intervals

path $a$ - Effects of perceived stigma on psychosocial factors,

path $b$ - Effects of psychosocial factor on suicidal ideation,

path $c^{\prime}$ - Direct effects of perceived stigma on suicidal ideation

path ab - Indirect effects of perceived stigma on suicidal ideation

external and objective stressors. We found that perceived stigma nonetheless had an impact on psychological processes among the TGW in our study. The implication of this conceptualization is that perceptions of the societal anti-transgender attitudes and structural level anti-transgender polices are important to assess even if they are considered as proxies for distal objective measures of stressors and that, for some TGW, perceptions are important to their mental health. TGW who perceive transgender-related stigma in our study, experienced increased psychosocial effects that could eventually be manifested in behaviors such as suicidal ideation.

\section{Limitations}

Although this study fills an important gap in the literature, several limitations exist. First, our study is a crosssectional study, and we cannot infer causation between predictor variables and suicidal ideation. In particular, the lack of temporality limits the interpretation of the findings from the mediation model. Future studies should investigate these associations among TGW in a longitudinal framework. In addition, convenience sampling was utilized to recruit participants, and most of the participants were referred through the community-based organization that provides support services to TGW. Therefore, our sample may not be representative of TGW who live in Atlanta. Due to the small sample size, this study may not have adequate statistical power to detect some significant associations. Finally, although culturally competent interviewers were utilized, social desirability bias may have affected some of the sensitive responses from participants. Recall bias also may have affected the participants' responses, particularly with regard to questions about early childhood.

\section{Conclusions}

TGW are disproportionately affected by suicidal ideation and are in urgent need of tailored and effective interventions to ameliorate their mental health concerns. Interventions that increase social inclusion may be particularly beneficial [13]. In one intervention utilizing mHealth to promote social support among TGW, a significant decrease in depressive symptoms and an increase in social inclusion was observed [56]. Moreover, policy-level interventions that aim at decreasing stigma, discrimination, and transphobia are likely to positively impact transgender individuals [13]. Education on gender diversity may also mitigate transphobia and raise awareness on gender identity [40]. Specifically, clinicians and psychologists should be culturally competent in the psychological issues and gender identity specific to TGW [41]. Clinicians should also use Minority Stress Theory as a framework for assessing and treating their gender minority patients. They also need to consider how experiences and perceptions of stigma and discrimination are chronic stressors that TGW must cope with and ultimately may manifest in internalized processes that contribute to risk behaviors and negative mental health outcomes $[41,57]$. Further, clinicians should support resiliency factors that are incorporated in minority stress theory, including group social support and encourage connecting to sexual and gender minority communities. Individual-level change also is critical to address the internalized stigma and trans-negativity among TGW. Based on the high levels of internalized stigma and the strong association with negative mental health outcomes, mental health professionals should work with TGW to address trans-negativity and provide healthy coping skills [40]. It is clear that structural-level interventions that seek to reduce stigma and 
discrimination and may have an effect on TGW's perceptions and experiences are greatly needed. By changing societal attitudes, including those of healthcare providers and structures (e.g., clinics, systems, etc.), ultimately, negative psychosocial impacts will be lessened, and TGW will expereience improved access, engagement, quality of care, and mental health outcomes (including decreased risk of suicide). Future research should focus on creating and evaluating multilevel interventions to help reduce suicidal ideation among TGW as well as evaluating the effectiveness of treatment that is based on the minority stress framework to clinical practice.

\section{Abbreviations \\ AOR: Adjusted Odds Ratio; CBO: Community Based Organization; Cl: Confidence Intervals; LGBT: Lesbian Gay Bisexual, and Transgender; MSM: Men who have sex with men; OR: Odds Ratio; TGW: Transgender women}

\section{Acknowledgements}

We thank our community partners who assisted greatly with our recruitment efforts.

\section{Authors' contributions}

KK was involved in data collection, data analysis, interpretation of results, and writing the manuscript. LFS was involved in designing the study, interpretation, and writing and reviewing the manuscript. REC was involved in data analysis, interpretation, and reviewing the manuscript. RAC was involved in designing the study, interpretation, and reviewing the manuscript. JJ was involved in data collection and reviewing the manuscript. All authors read and approved the final manuscript.

\section{Funding}

Funding was provided, in part, by the fourth author's Good Samaritan Endowment. The funding source had no role in study design, data collection, analysis, data interpretation, writing of the report; and in the decision to submit the paper for publication.

\section{Availability of data and materials}

The datasets analyzed during the current study are not publicly available as it is a small pilot study and contain information that could compromise research participant privacy but are available from the corresponding author on reasonable request.

\section{Ethics approval and consent to participate}

The Institutional Review Board of Georgia State University approved study protocols following a full board review. Signed Informed Consents were obtained from the study participants.

\section{Consent for publication}

Not Applicable.

\section{Competing interests}

The authors declare that they have no competing interests.

\section{Author details}

'Department of Health Policy \& Behavioral Science, School of Public Health Georgia State University, PO Box 3984, Atlanta, GA 30302-3984, USA.

${ }^{2}$ Department of Respiratory Therapy, Byrdine F. Lewis College of Nursing and Health Professions, Georgia State University, Atlanta, GA, USA. ${ }^{3}$ Department of Health Promotion, College of Public Health, University of Kentucky, Lexington, KY, USA. ${ }^{4}$ Kinsey Institute for Research on Sex, Gender, and Reproduction, Indiana University, Bloomington, IN, USA.
Received: 14 February 2019 Accepted: 8 January 2020

Published online: 29 January 2020

\section{References}

1. Bockting WO. Psychotherapy and the real-life experience: from gender dichotomy to gender diversity. Sexologies. 2008;17(4):211-24.

2. Bockting WO, et al. Evaluation of a sexual health approach to reducing HIV/ STD risk in the transgender community. AIDS Care. 2005;17(3):289-303.

3. Wo B. From construction to context: gender through the eyes of the transgendered. SIECUS Rep. 1999;28(1):3.

4. Association, A.P. Report of the APA task force on gender identity and gender variance. Washington, DC: American Psychological Association; 2009

5. Grant JM, et al. Injustice at every turn: a report of the National Transgender Discrimination Survey. Washington: National Center for Transgender Equality and National Gay and Lesbian Task Force; 2011.

6. Marshall $E$, et al. Non-suicidal self-injury and suicidality in trans people: a systematic review of the literature. Int Rev Psychiatry. 2016;28(1):58-69.

7. Mustanski B, Liu RT. A longitudinal study of predictors of suicide attempts among lesbian, gay, bisexual, and transgender youth. Arch Sex Behav. 2013; 42(3):437-48.

8. James SE, Herman JL, Rankin S, Keisling M, Mottet L, Anafi M. The report of the 2015 U.S. transgender survey. Washington, DC: National Center for transgender equality; 2016

9. Testa RJ, et al. Suicidal ideation in transgender people: gender minority stress and interpersonal theory factors. J Abnorm Psychol. 2017;126(1): $125-36$

10. Perez-Brumer A, et al. Individual- and structural-level risk factors for suicide attempts among transgender adults. Behav Med. 2015:41(3):164-71.

11. Crosby AE, O. L, Melanson C. Self-directed violence surveillance: uniform definitions and recommended data elements. Atlanta: Centers for Disease Control and Prevention, National Center for injury prevention and Control; 2011.

12. Grossman AH, D'Augelli AR. Transgender youth and life-threatening behaviors. Suicide Life Threat Behav. 2007;37(5):527-37.

13. Bauer $G R$, et al. Intervenable factors associated with suicide risk in transgender persons: a respondent driven sampling study in Ontario, Canada. BMC Public Health. 2015:15:525.

14. Tebbe EA, Moradi B. Suicide risk in trans populations: an application of minority stress theory. J Couns Psychol. 2016:63(5):520-33.

15. Adams N, Hitomi M, Moody C. Varied reports of adult transgender suicidality: synthesizing and describing the peer-reviewed and gray literature. Transgend Health. 2017;2(1):60-75

16. Brown GK. A review of suicide assessment measures for intervention research in adults and older adults. Bethesda: National Institute of Mental Health; 2002.

17. Ahrens B, et al. Suicidal behavior--symptom or disorder? Compr Psychiatry. 2000;41(2 Suppl 1):116-21.

18. Oquendo MA, et al. Sex differences in clinical predictors of suicidal acts after major depression: a prospective study. Am J Psychiatry. 2007;164(1):134-41.

19. De Leo $D$, et al. Repetition of suicidal behaviour in elderly Europeans: a prospective longitudinal study. J Affect Disord. 2002;72(3):291-5.

20. Center NLHE. Glossary of LGBT terms for health care teams. Boston: The Fenway Institute; 2017

21. May AM, Klonsky ED, Klein DN. Predicting future suicide attempts among depressed suicide ideators: a 10-year longitudinal study. J Psychiatr Res. 2012:46(7):946-52

22. Han B, McKeon R, Groerer J. Suicidal ideation among community-dwelling adults in the United States. Am J Public Health. 2014;104(3):488-97.

23. Nock MK, et al. Mental disorders, comorbidity and suicidal behavior: results from the National Comorbidity Survey Replication. Mol Psychiatry. 2010; 15(8):868-76.

24. Vanderwerker $L C$, et al. Differences in risk factors for suicidality between African American and White patients vulnerable to suicide. Suicide Life Threat Behav. 2007;37(1):1-9.

25. Clements-Nolle K, Marx R, Katz M. Attempted suicide among transgender persons: the influence of gender-based discrimination and victimization. J Homosex. 2006:51(3):53-69.

26. Haas A, Rodgers P, Herman J. Suicide Attempts Among Transgender and Gender Non-Conforming Adults: finding of the National Transgender Discrimination Survey; 2014. 
27. Klein A, Golub SA. Family rejection as a predictor of suicide attempts and substance misuse among transgender and gender nonconforming adults. LGBT Health. 2016;3(3):193-9.

28. Valentine SE, Shipherd JC. A systematic review of social stress and mental health among transgender and gender non-conforming people in the United States. Clin Psychol Rev. 2018;66:24-38.

29. Operario D, et al. Stigma and the syndemic of HIV-related health risk behaviors in a diverse sample of transgender women. J Community Psychol. 2014;42(5):544-57.

30. White Hughto JM, Reisner SL, Pachankis JE. Transgender stigma and health: a critical review of stigma determinants, mechanisms, and interventions. Soc Sci Med. 2015;147:222-31.

31. Nemoto T, Bödeker B, Iwamoto M. Social support, exposure to violence and transphobia, and correlates of depression among male-to-female transgender women with a history of sex work. Am J Public Health. 2011; 101(10):1980-8.

32. Reisner SL, et al. Legal protections in public accommodations settings: a critical public health issue for transgender and gender-nonconforming people. Milbank Q. 2015;93(3):484-515.

33. Bockting WO, et al. Stigma, mental health, and resilience in an online sample of the US transgender population. Am J Public Health. 2013; 103(5):943-51

34. Gamarel KE, et al. Gender minority stress, mental health, and relationship quality: a dyadic investigation of transgender women and their cisgender male partners. J Fam Psychol. 2014;28(4):437-47.

35. Gamarel KE, et al. Association between socioeconomic position discrimination and psychological distress: findings from a community-based sample of gay and bisexual men in new York City. Am J Public Health. 2012; 102(11):2094-101.

36. Yang MF, et al. Stigmatization and mental health in a diverse sample of transgender women. LGBT Health. 2015;2(4):306-12.

37. Veale JF, et al. Enacted stigma, mental health, and protective factors among transgender youth in Canada. Transgend Health. 2017;2(1):207-16.

38. Meyer IH. Prejudice, social stress, and mental health in lesbian, gay, and bisexual populations: conceptual issues and research evidence. Psychol Bull. 2003;129(5):674-97.

39. Hatzenbuehler ML. How does sexual minority stigma "get under the skin"? A psychological mediation framework. Psychol Bull. 2009;135(5):707-30.

40. Staples JM, et al. The role of distal minority stress and internalized Transnegativity in suicidal ideation and nonsuicidal self-injury among transgender adults. J Sex Res. 2018;55(4-5):591-603.

41. Hendricks ML, Testa RJ. A conceptual framework for clinical work with transgender and gender nonconforming clients: an adaptation of the minority stress model. Prof Psychol Res Pract. 2012;43(5):460-7.

42. Scandurra $C$, et al. Internalized transphobia, resilience, and mental health: Applying the psychological mediation framework to Italian transgender individuals. Int J Environ Res Public Health. 2018;15(3):508.

43. Mereish EH, O'Cleirigh C, Bradford JB. Interrelationships between LGBT-based victimization, suicide, and substance use problems in a diverse sample of sexual and gender minorities. Psychol Health Med. 2014;19(1):1-13.

44. Ross MW, Rosser BR. Measurement and correlates of internalized homophobia: a factor analytic study. J Clin Psychol. 1996;52(1):15-21.

45. Smolenski DJ, et al. Revision, criterion validity, and multigroup assessment of the reactions to homosexuality scale. J Pers Assess. 2010;92(6):568-76.

46. Sjoberg MD, Walch SE, Stanny CJ. Development and initial psychometric evaluation of the transgender adaptation and integration measure (TG AIM). Int J Transgend. 2006;9(2):35-45.

47. Derogatis LR. Brief symptom inventory (BSI) 18: administration, scoring, and procedures manual. Minneapolis: NCS Pearson; 2000

48. DHHS. Dietary Guidelines for Americans, 2015-2020. T.U.S.D.o.H.a.H. services, Editor; 2015.

49. Mackinnon DP, Dwyer JH. Estimating mediated effects in prevention studies. Eval Rev. 1993;17(2):144-58.

50. Preacher KJ, Hayes AF. Asymptotic and resampling strategies for assessing and comparing indirect effects in multiple mediator models. Behav Res Methods. 2008;40(3):879-91.

51. Hayes AF. Introduction to mediation, moderation, and conditional process analysis: A regression-based approach. Methodology in the social sciences, Guilford Press; 2013.
52. Preacher KJ, Kelley K. Effect size measures for mediation models: quantitative strategies for communicating indirect effects. Psychol Methods. 2011;16(2):93-115.

53. Perez-Brumer A, et al. Prevalence and correlates of suicidal ideation among transgender youth in California: findings from a representative, populationbased sample of high school students. J Am Acad Child Adolesc Psychiatry. 2017;56(9):739-46.

54. Factor RJ, Rothblum ED. A study of transgender adults and their nontransgender siblings on demographic characteristics, social support, and experiences of violence. J LGBT Health Res. 2007;3(3):11-30.

55. Gotink RA, et al. Standardised mindfulness-based interventions in healthcare: an overview of systematic reviews and meta-analyses of RCTs. PLoS One. 2015;10(4):e0124344.

56. Hightow-Weidman LB, et al. HealthMpowerment.Org: building community through a Mobile-optimized, online health promotion intervention. Health Educ Behav. 2015;42(4):493-9.

57. Alessi EJ. A framework for incorporating minority stress theory into treatment with sexual minority clients. J Gay Lesbian Mental Health. 2014; 18(1):47-66

\section{Publisher's Note}

Springer Nature remains neutral with regard to jurisdictional claims in published maps and institutional affiliations.
Ready to submit your research? Choose BMC and benefit from:

- fast, convenient online submission

- thorough peer review by experienced researchers in your field

- rapid publication on acceptance

- support for research data, including large and complex data types

- gold Open Access which fosters wider collaboration and increased citations

- maximum visibility for your research: over $100 \mathrm{M}$ website views per year

At BMC, research is always in progress.

Learn more biomedcentral.com/submissions 